\title{
Hemorrhagic pancreatic cyst in living donor liver transplantation: a case report
}

\author{
Tae Yun Lee, Young Chul Yoon
}

Department of Surgery-Hepatobiliary, The Catholic University of Korea, Incheon St. Mary's Hospital, Incheon, Korea

Background: A 27-year-old male, a daily alcohol drinker, was admitted to our hospital for abdominal distension and jaundice that had occurred 1 month before admission.

Case report: In computed tomography (CT) imaging, the patient was alcoholic liver cirrhosis with uneven fatty liver and splenomegaly. He had a $2.6-\mathrm{cm}$ sized cystic lesion with thick enhancing wall at peripancreatic space. After about 3 weeks, preoperative magnetic resonance imaging showed an enlarged cyst from $2.6 \mathrm{~cm}$ to $4.1 \mathrm{~cm}$. Ten days later, he received living donor liver transplantation (LDLT) with a liver donor from his father, and the graft-to-recipient weight ratio was $0.75 \%$, which was a concern for the small for size syndrome. Since liver function did not recover after liver transplantation, an early CT scan was performed. As a result of imaging, splenic artery steel syndrome was suspected. Angiography was immediately performed, and splenic artery embolization was performed. Also, this CT showed that the cyst in the tail of the pancreas increased significantly from $4.1 \mathrm{~cm}$ to $8.1 \mathrm{~cm}$. Two weeks later, the patient complained of severe abdominal pain, and CT showed actively bleeding pseudocyst from pancreas tail, showing increased size $(11.2 \mathrm{~cm}$ from $9.2 \mathrm{~cm})$. Emergency re-operation was performed. Distal pancreatectomy with splenectomy and proximal gastrectomy surgery was performed. Even after surgery, the patient did not recover liver function and eventually expired.

Conclusions: Usually, pancreatic cysts before and after liver transplantation are known to be harmless. However, in view of the above case, close observation of pancreatic cyst during $L T$ is considered necessary.

Corresponding author: Young Chul Yoon

E-mail:k07yyc@catholic.ac.kr

(c) The Korean Society for Transplantation

This is an Open Access article distributed under the terms of the Creative Commons Attribution Non-Commercial License (http://creativecommons.org/licenses/by-nc/4.0/) which permits unrestricted non-commercial use, distribution, and reproduction in any medium, provided the original work is properly cited. 